\title{
Identification of a small, depressed type of colorectal invasive cancer by an artificial intelligence-assisted detection system
}

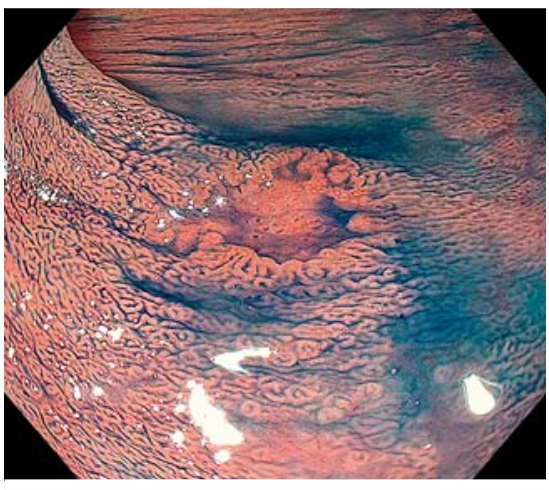

- Fig. 1 Spraying of indigo carmine revealed a clear depression (Paris 0 -Ilc lesion).

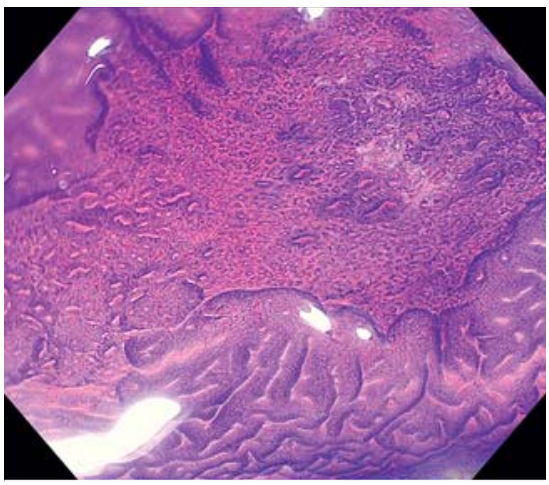

> Fig. 2 Crystal-violet dye staining with magnification, showing irregular-shaped pit patterns of varying sizes (Kudo's type $V_{1}$ pit pattern).

A 64-year-old man underwent surveillance colonoscopy with a computer-aided detection (CADe) system (EndoBRAIN-EYE; Cybernet Systems, Tokyo, Japan) [1]. The system identified a 5-mm slightly reddish lesion in the sigmoid colon. Spraying with indigo carmine enabled identification of a clearly depressed area on the lesion ( $\triangleright$ Fig. 1, $\triangleright$ Video 1 ). The lesion showed type $V_{\text {I }}$ pit pattern, indicating high grade dysplasia or slightly invasive submucosal cancer [2]. Endoscopic mucosal resection was performed. Pathological examination showed a well-differentiated adenocarcinoma with
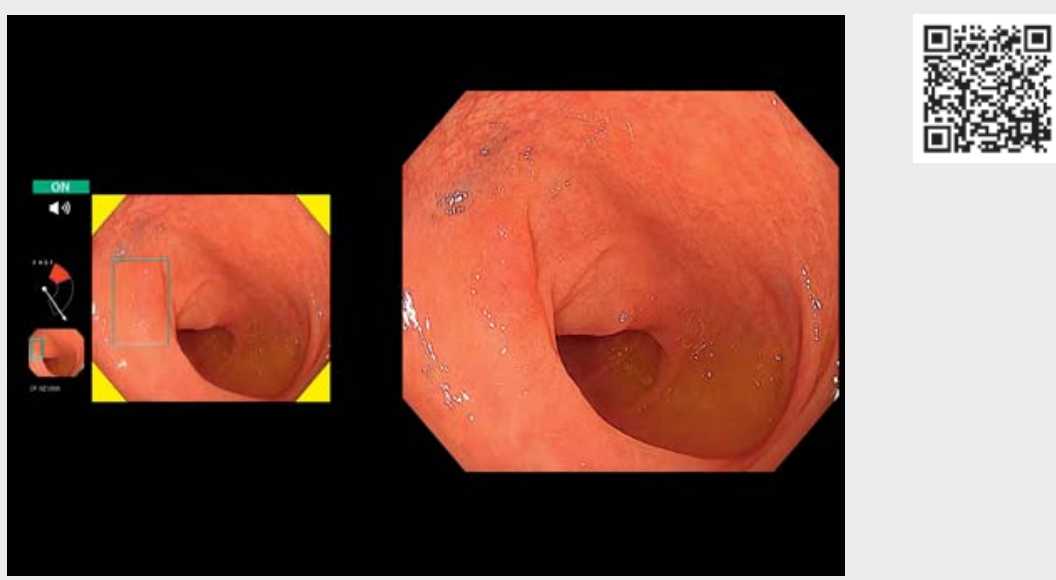

Video 1 The EndoBRAIN-EYE (Cybernet Systems, Tokyo, Japan) outputs bounding boxes of suspected polyp candidate areas. The left image is the system's output, and the right image is the original endoscopic image.

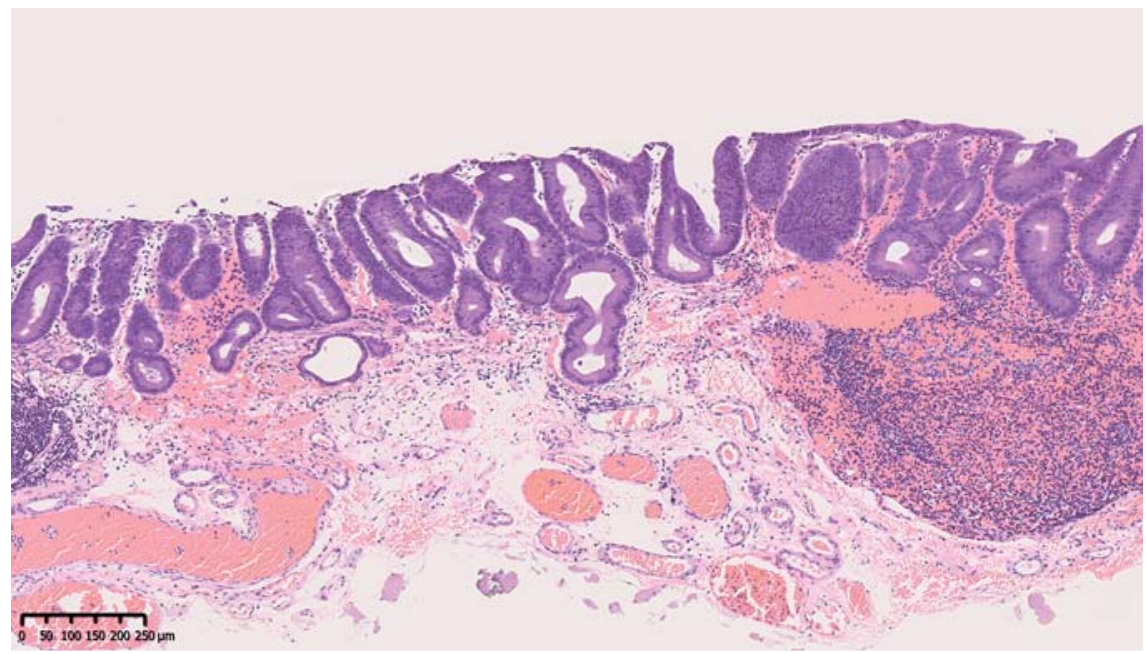

- Fig. 3 Photomicrograph of the specimen (hematoxylin and eosin staining), showing a welldifferentiated adenocarcinoma.

slight invasion of the submucosal layer ( $\triangleright$ Fig. 2, $\triangleright$ Fig. 3, $>$ Fig. 4).

Artificial intelligence (AI) technology has regulatory clearance and is increasingly used during colonoscopy. A meta-analysis showed that CADe systems increase adenoma detection rates [3]. However, identifying subtle nonpolypoid lesions (e.g. 0-Ilc type depressed lesions; laterally spreading tumors without granules) with CADe is still considered challenging. This is clinically relevant because a recent randomized trial found that such nonpolypoid tumors may be one of the causes of post-colonoscopy colorectal cancer [4]. Such lesions have greater 


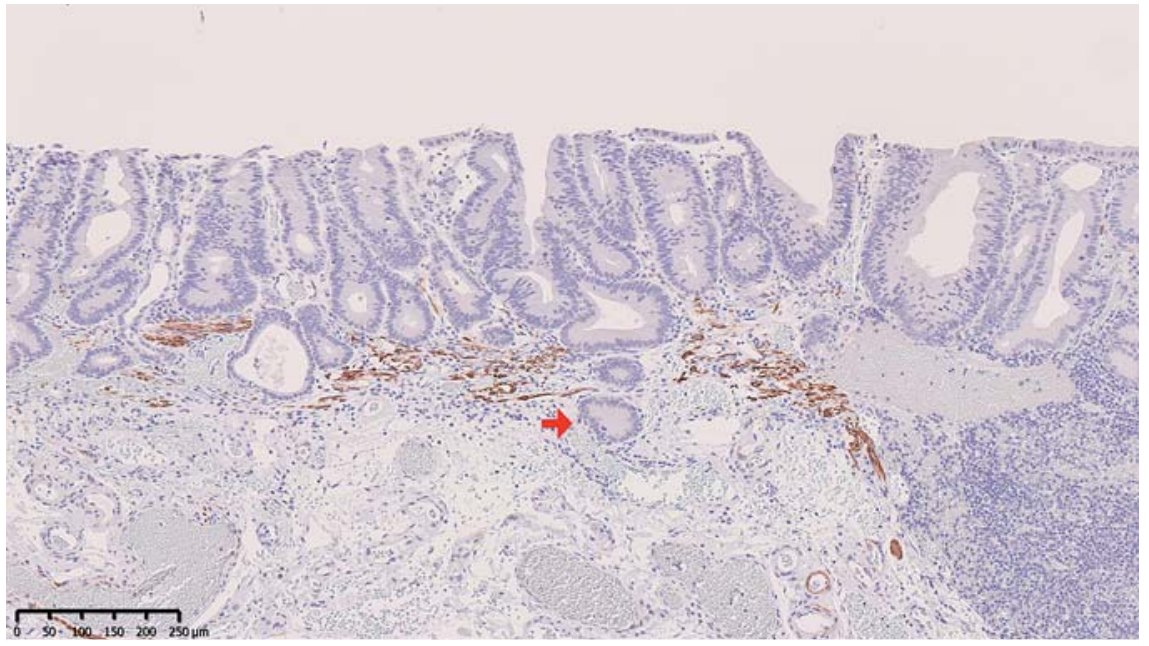

- Fig. 4 Photomicrograph showing that one cancerous gland (red arrow) invaded the submucosal layer beyond the muscularis mucosa (desmin immunostaining).

malignant potential than other tumor morphologies and are often overlooked because of their appearance [5]. To the best of our knowledge, this is the first report of detection of a depressed, type 0 -llc lesion by CADe in real time during clinical colonoscopy. This Al-assisted detection was of particular value because the lesion was found to be a submucosally invasive colorectal cancer.

\section{Endoscopy_UCTN_Code_TTT_1AQ_2AB}

\section{Acknowledgment}

We thank Dr. Trish Reynolds, MBBS, FRACP, from Edanz (https://jp.edanz.com/ac) for editing a draft of this manuscript.

\section{Competing interests}

Shin-ei Kudo, Masashi Misawa, and Yuichi Mori have received speaking honoraria from Olympus Corporation (Tokyo, Japan) and have ownership interest in the products of Cybernet Systems (Tokyo, Japan). Masashi Misawa, Shin-ei Kudo, and Yuichi Mori have patents (Japan Patent JP 6059271 and JP 6580446) licensed to Cybernet Systems and Showa University.
The authors

Shin-ei Kudo ${ }^{1}$, Masashi Misawa ${ }^{1}$, Yuichi

Mori $^{1,2}{ }^{2}$, Yurie Kawabata ${ }^{1}$, Yasuharu

Maeda $^{1}{ }^{\oplus}$, Hideyuki Miyachi' ${ }^{1}$, Kensaku Mori ${ }^{3}$

1 Digestive Disease Center, Showa University Northern Yokohama Hospital, Japan

2 Clinical Effectiveness Research Group. Institute of Health and Society, Faculty of Medicine, University of Oslo, Oslo, Norway

3 Graduate School of Informatics, Nagoya University, Nagoya, Japan

\section{Corresponding author}

\section{Shin-ei Kudo, MD, PhD}

Digestive Disease Center, Showa University, Showa University Northern Yokohama Hospital, 35-1 Chigasaki-chuo, Tsuzuki, Yokohama, 224-8503, Japan kudos@med.showa-u.ac.jp

\section{References}

[1] Ishiyama M, Kudo SE, Misawa M et al. Impact of the clinical use of artificial intelligenceassisted neoplasia detection for colonoscopy: a large-scale prospective, propensity score-matched study (with video). Gastro- intest Endosc 2021. doi:10.1016/j. gie.2021.07.022

[2] Kudo SE, Rubio CA, Teixeira CR et al. Pit pattern in colorectal neoplasia: endoscopic magnifying view. Endoscopy 2001; 33: $367-$ 373

[3] Barua I, Vinsard DG, Jodal HC et al. Artificial intelligence for polyp detection during colonoscopy: a systematic review and metaanalysis. Endoscopy 2021; 53: 277-284

[4] Matsuda T, Fujii T, Sano Y et al. Randomised comparison of postpolypectomy surveillance intervals following a two-round baseline colonoscopy: the Japan Polyp Study Workgroup. Gut 2020; 70: 1469-1478

[5] Soetikno RM, Kaltenbach T, Rouse RV et al. Prevalence of nonpolypoid (flat and depressed) colorectal neoplasms in asymptomatic and symptomatic adults. JAMA 2008; 299: 1027-1035

Bibliography

Endoscopy 2022; 54: E592-E593

DOI 10.1055/a-1704-8103

ISSN 0013-726X

published online 21.12.2021

(c) 2021. Thieme. All rights reserved.

Georg Thieme Verlag KG, Rüdigerstraße 14,

70469 Stuttgart, Germany

\section{ENDOSCOPY E-VIDEOS}

https:/|eref.thieme.de/e-videos

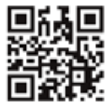

Endoscopy E-Videos is an open access online section, reporting on interesting cases and new techniques in gastroenterological endoscopy. All papers include a high quality video and all contributions are freely accessible online. Processing charges apply (currently EUR 375), discounts and wavers acc. to HINARI are available.

This section has its own submission website at https://mc.manuscriptcentral.com/e-videos 